\title{
شبكات التواصل الاجتماعي بين اختصاص البنية وعمومية الاستعمال مدخل نظري
}

$$
\begin{aligned}
& \text { شراد سهيل } \\
& \text { كلية علوم الإعــلام و الاتصـال والسمعي }
\end{aligned}
$$

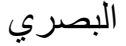

$$
\begin{aligned}
& \text { جامعة صالح بوبنيدر - قسنطينة- 3- }
\end{aligned}
$$

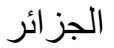

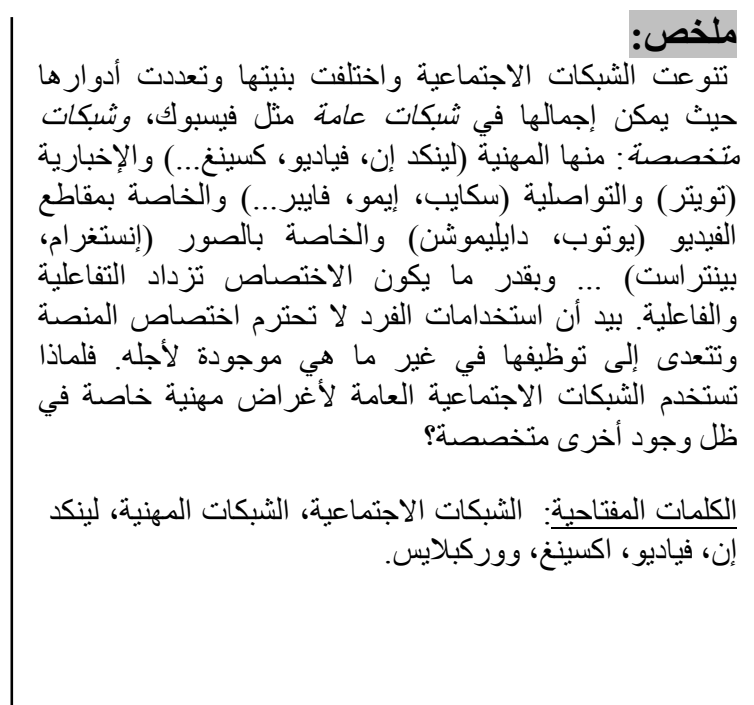

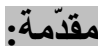

لم تلتوقف يوما محاو لات

الإنسان لتطوير الأدوات التي لتوفي

يستخدمها للتعبير عن آرائه

وأفكاره و إعلام المحيطين بها

وكذلك الأدو ات اللازمة لتوثيق

خبر اته ومعارفه، كما لم تصل

رغبتنا الفطرية في التو اصل مع ومعاده

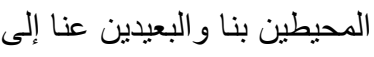

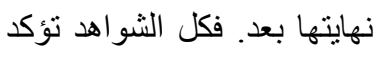

أن التطور في مجالات الإعلام

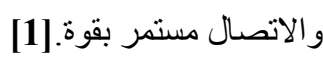

\begin{abstract}
:
Social networks have varied and diversified in structure and multiple in roles, which can be summarized in public networks allow individuals to present themselves and establish or maintain connections with others such as Facebook, and specialized networks: Professional, can be oriented towards work-related contexts ( LinkedIn, Viadeo, Xing ...), or informational by Sharing thoughts and keeping up with others via this real-time (e.g., Twitter ) or else communication like (Skype, Imo, Viber ), special contents as videos (YouTube, Dailymotion...) and photography (Instagram, Pintrest)... As far as it is specialized, the interactivity increases and efficiency. However, the uses of individuals do not respect the particularity of the platform and go beyond employing it in what it does not exist for. Why are public social networks used for special professional purposes where other specialized ones exist?
\end{abstract}

Key Word : Social Networks, Professional SNS, LinkedIn, Viadeo, Xing, WorkPlace.

(C) جامعة الإخوة منتوري قسنطينة، الجز ائر 
تزداد أهمية الإنترنيت مع تتوع استعمالاتها، وازدياد عدد المستخدمين لها، ولا تتحصر أهمية الانترنت

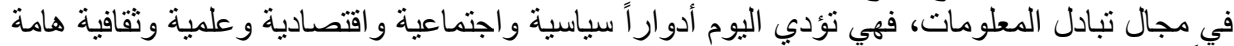

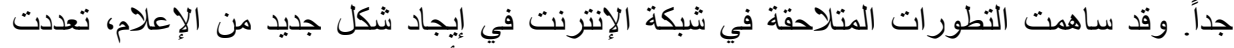

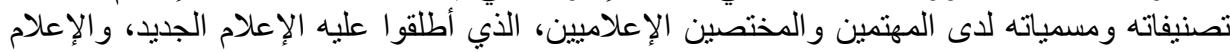

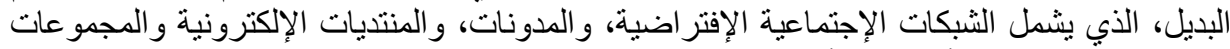

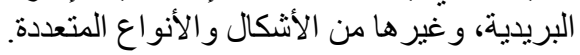

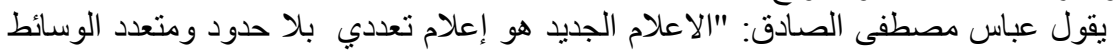

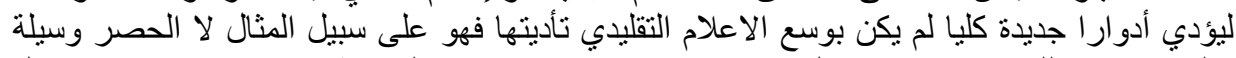

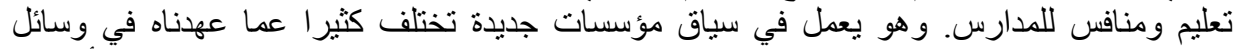

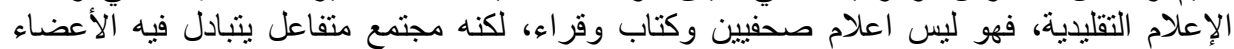

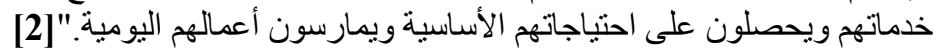

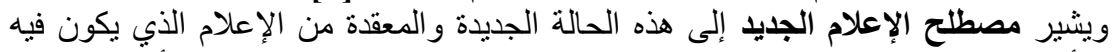

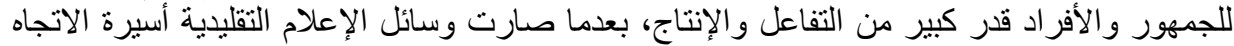

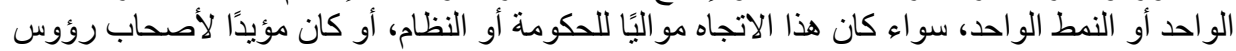

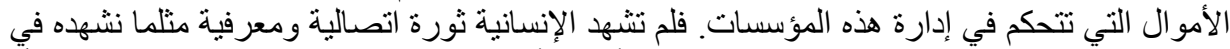

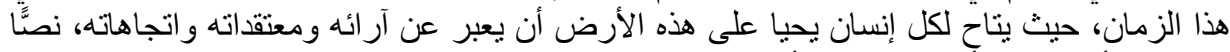

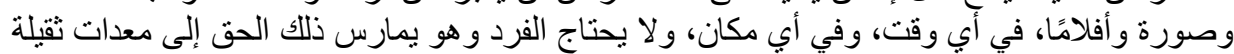

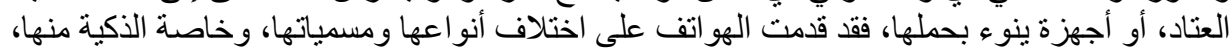

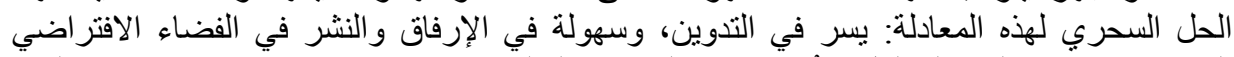

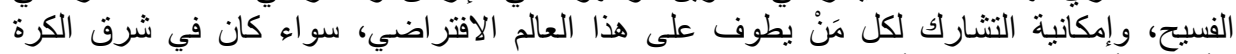

الأرضية أو غربها، شمالها أو جنوبها. [3:

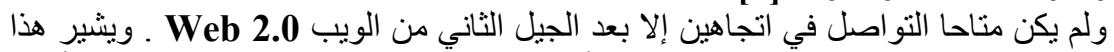

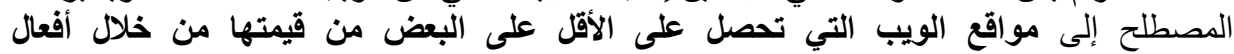

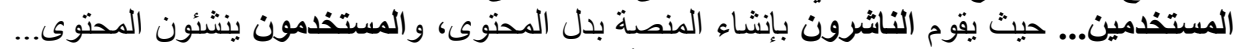

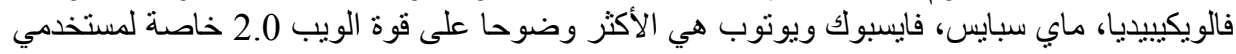

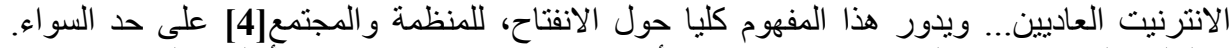

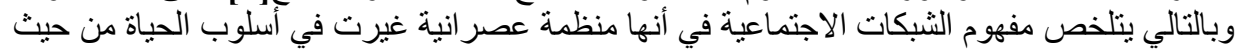

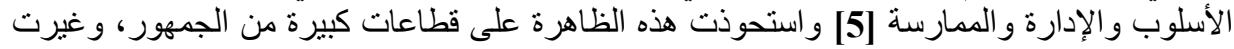
كثيرًا من ملامح البيئة الاتصالية.

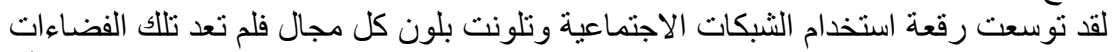

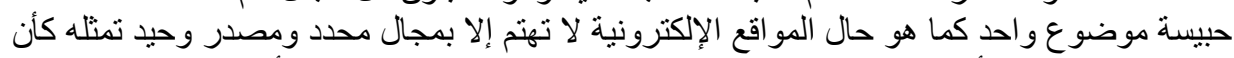

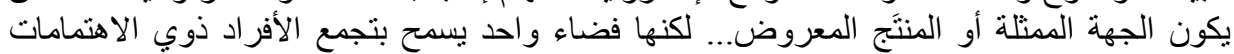

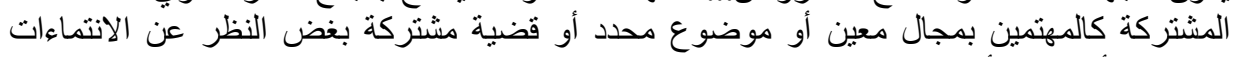

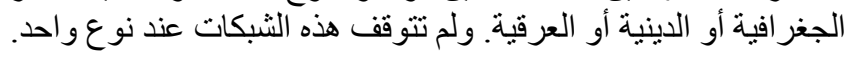
I I

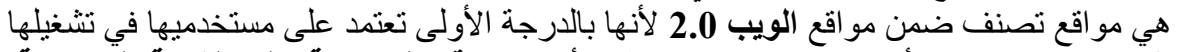

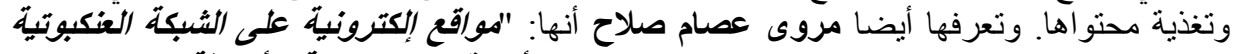

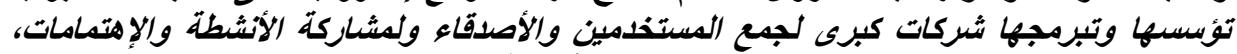

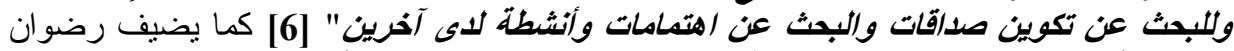

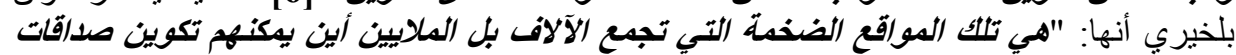

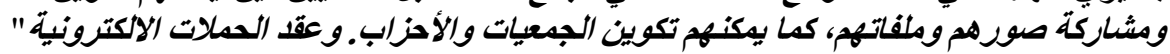




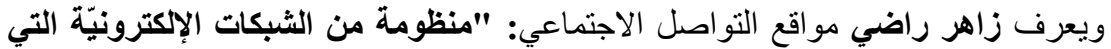

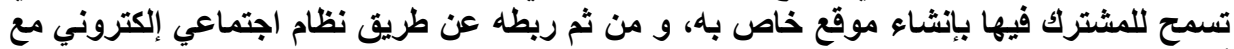

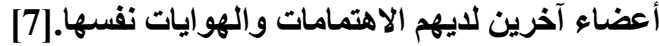

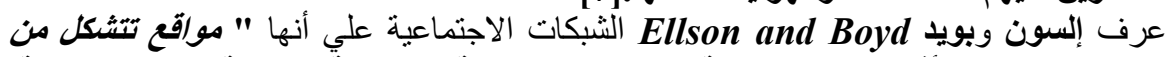

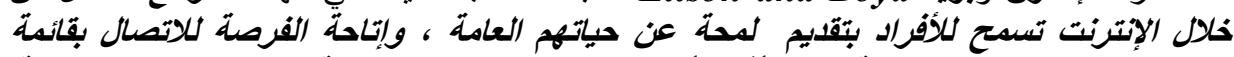

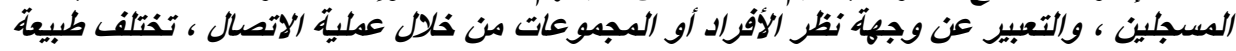

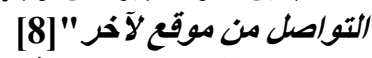
إذن هي مجموعة من المواقع الإلكترونية، الموجودة على شبكة الإنترنت، وهدفه الإنها الرئيسي

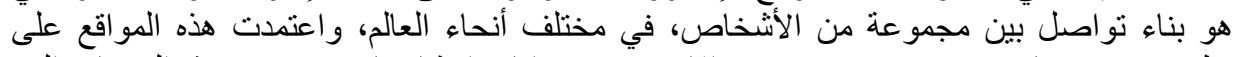

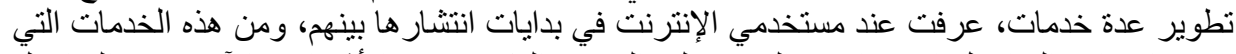

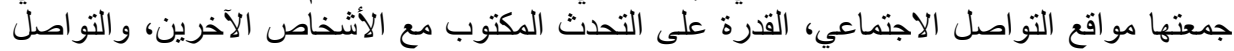

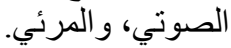

نشأة الشبكات الاجتماعية

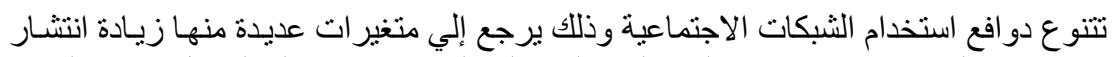
.III

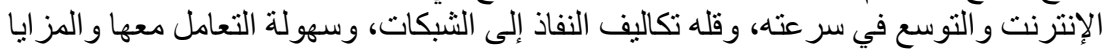

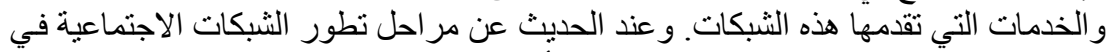

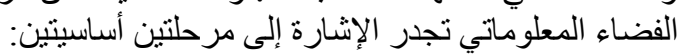

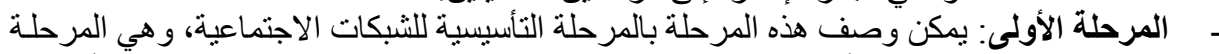

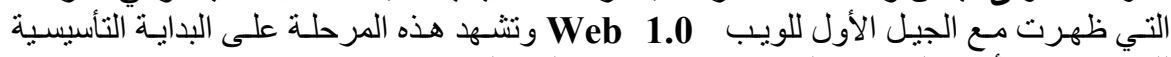

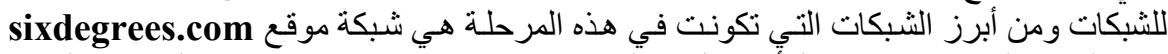

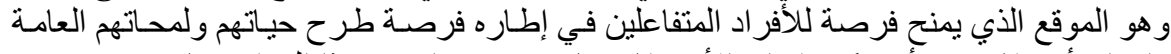

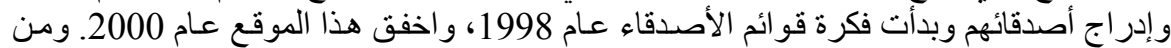

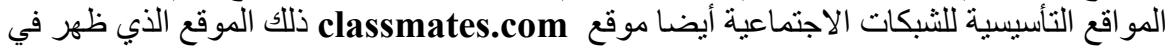

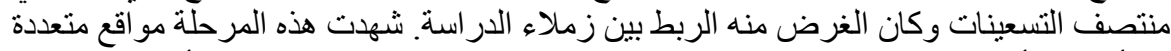

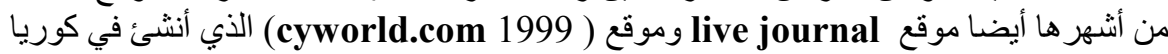

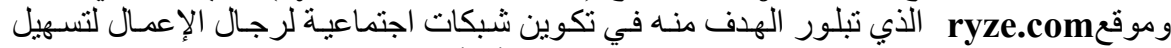

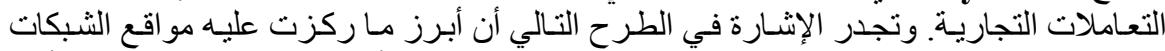

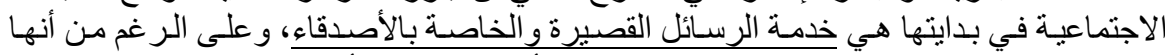

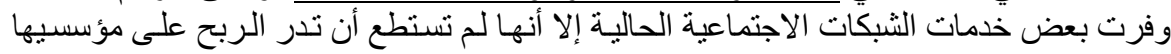
ولم يكتب لكثير منها البقاء.

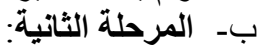

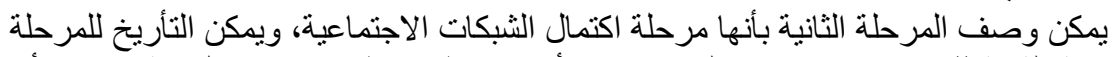

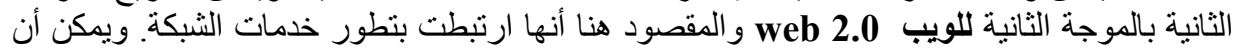

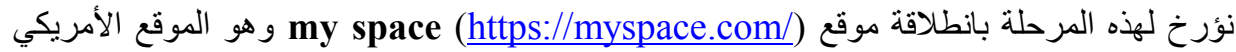

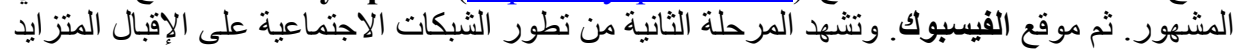

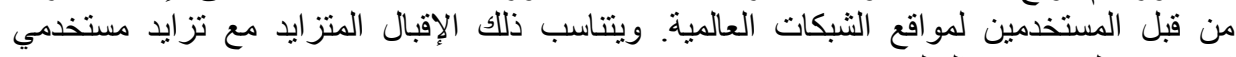

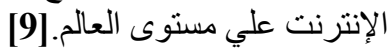
الخدمات

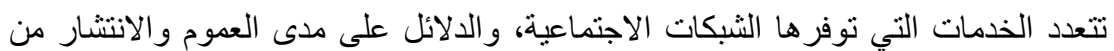

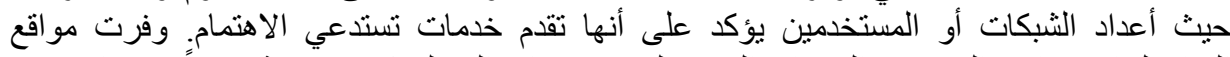
التو اصل الاجتماعي العديد من الخدمات الجديدة لمستخدميها، و التي لم تكن معروفة مسبقاً، ومنها: 
1-الملفات الشخصة (Profile): وهي ملفات تمكن من خلالها الفرد من كتابة بياناته

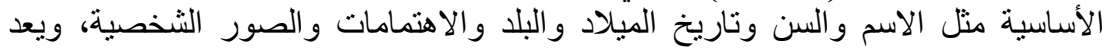

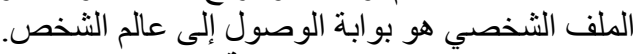

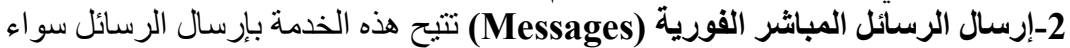

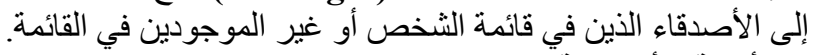

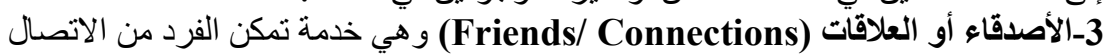

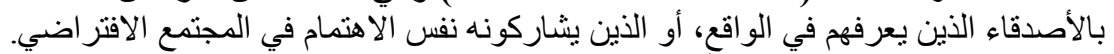

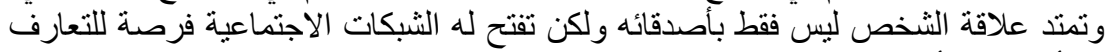
مع أصدقاء الأصدقاء بعد مو افقة الطرفين.

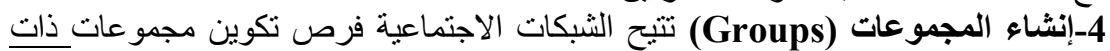

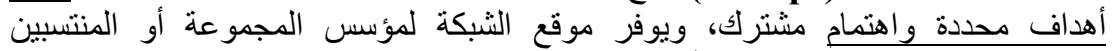

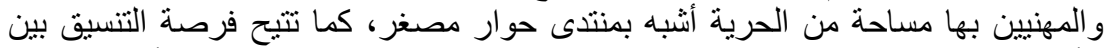

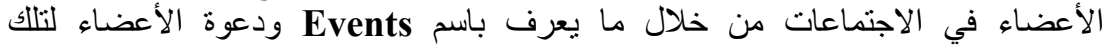

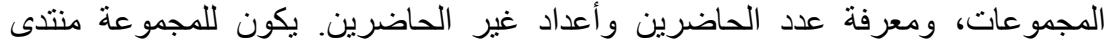

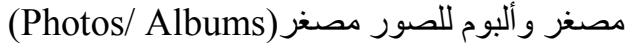

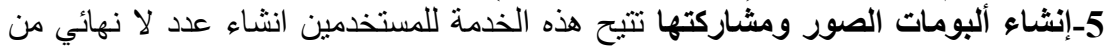

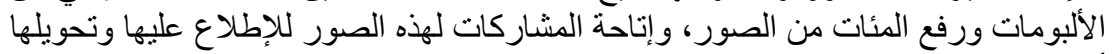

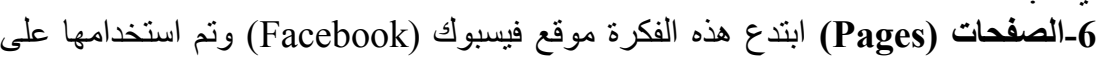

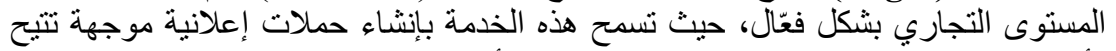

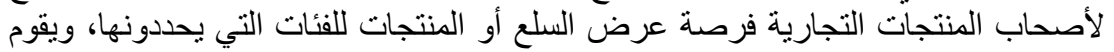

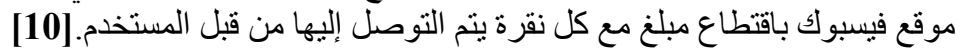

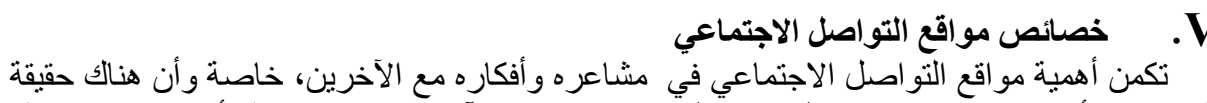

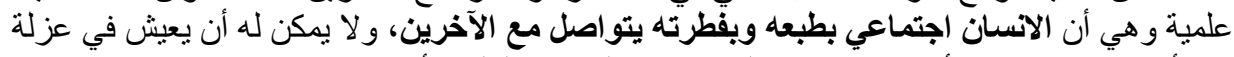

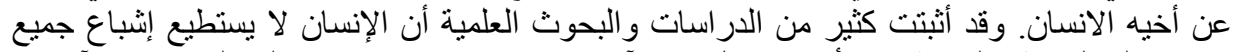

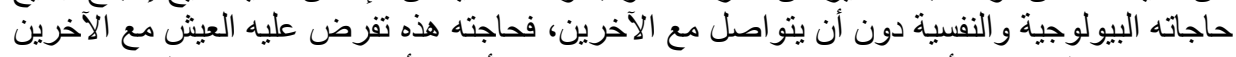

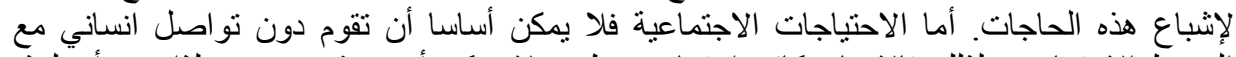

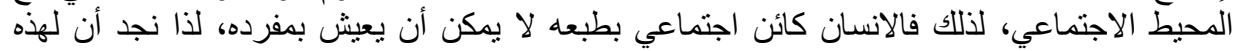

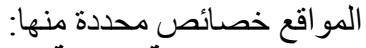

1. العالمية (شاملة ): حيث تلغي الحواجز الجغر افية و المكانية، تلغى من خلالها الحدود الدولية،

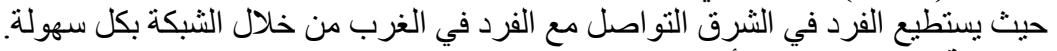

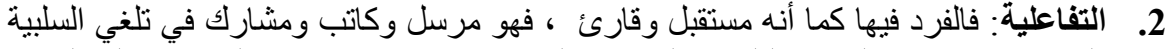

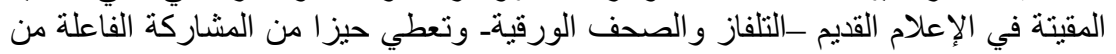

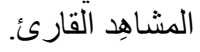

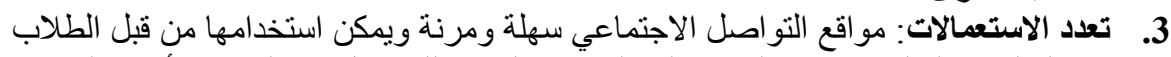

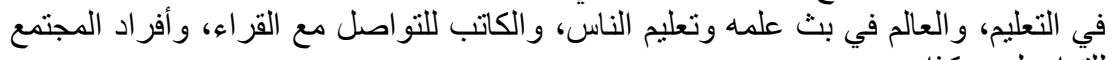
ل اللتو اصل وهكذا. 4. سهولة الاستخدام: فالثبكات الاجتماعية تستخدم بالإضافة إلى الحروف وبساطة اللغة، تستخدم الرموز و الصور التي تسهل للمستخدم نقل فكرته و التفاعل مع الآخرين. 


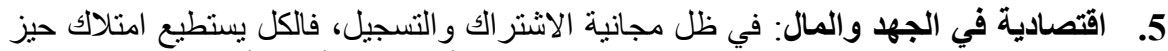

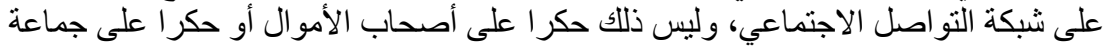

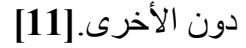

الاستعمالات في البيئة المهنية

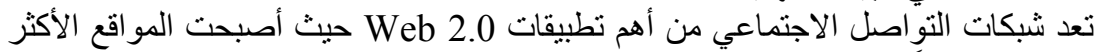

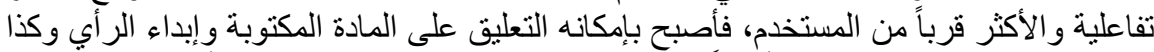

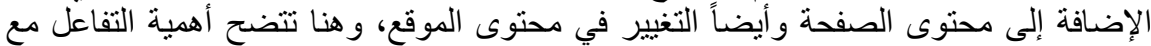

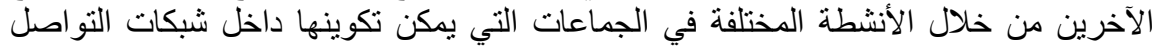

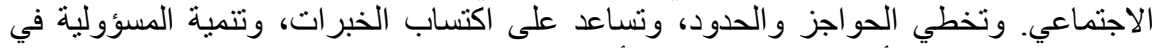

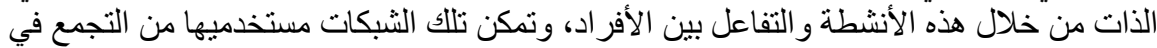

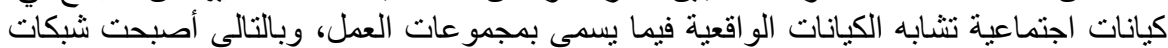

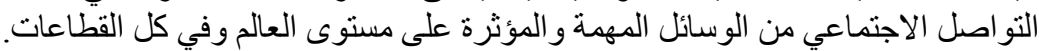

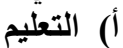

تلعب الثبكات الاجتماعية دورا في تعزيز العملية التعليمية التعلمية من خلال تطوير التعليم

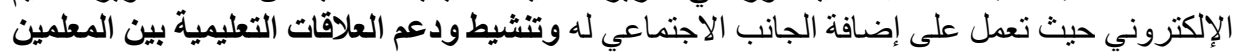

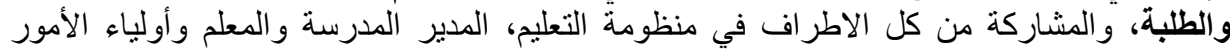

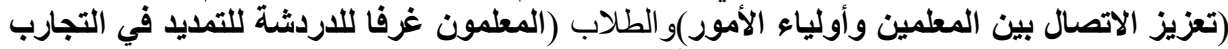

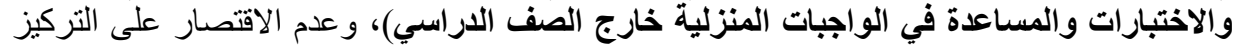

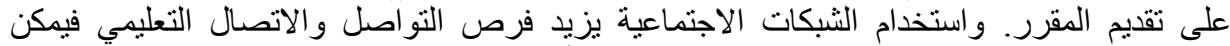

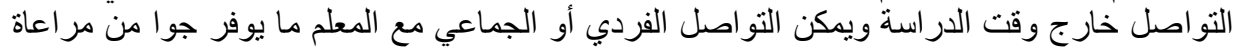

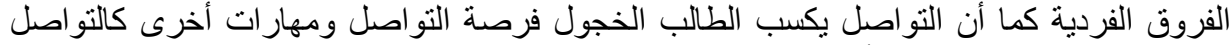

و الاتصال و المناقثة و ابداء الر أي. [12]

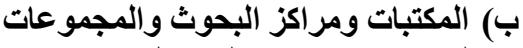

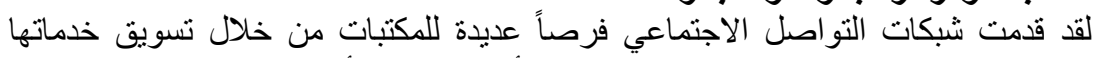

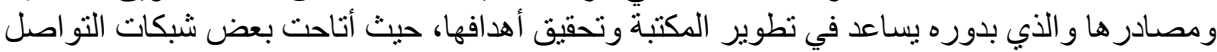

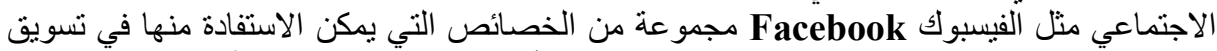

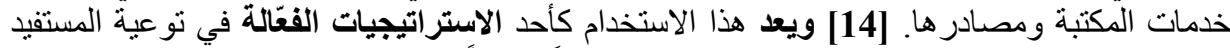

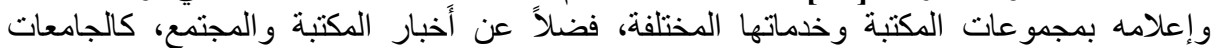

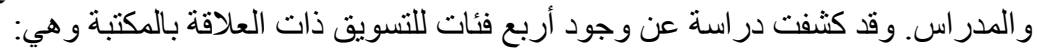

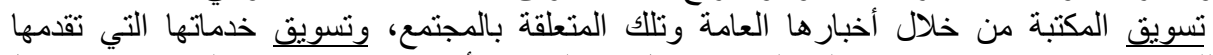

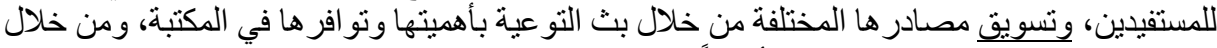

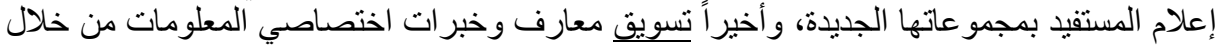
عرض مهار اته البحثية، وقدرنه على مساعدة المستفيدين، وذللك لحث المستفيدين على الاستفادة من التهن

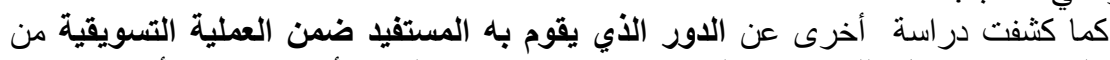
خبرات موظفي المكتبة.

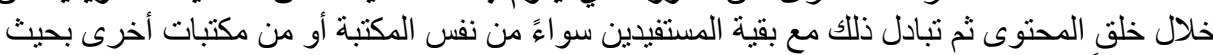

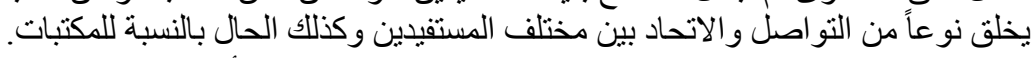

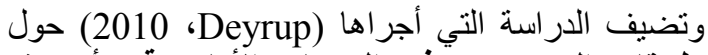

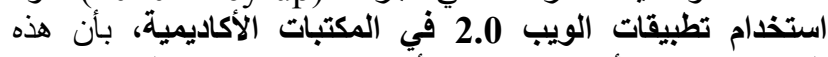

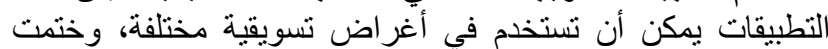

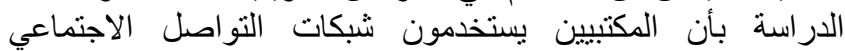
Facebook و MySpace لغرض تحسين سمعة المكتبة، والرقي الرئي

"الثبكات الاجتماعية أداة"

تعليم وتعلم" 
بالمجتمع من خلال عرض خدمات المكتبة و أنشطتها. [13]

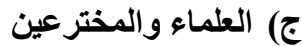

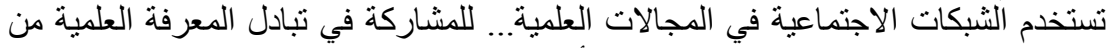

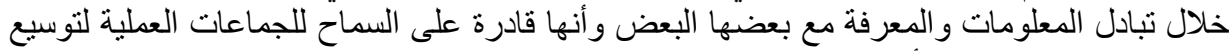

قاعدتها المعرفية وتبادل الأفكار . [15]

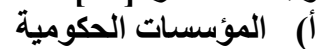

تمثل الثبكات الاجتماعية "طريقة سهلة وسريعة للحكومة للحصول على رلمات رأي الجمهور بهدف

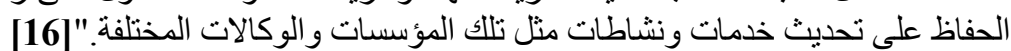

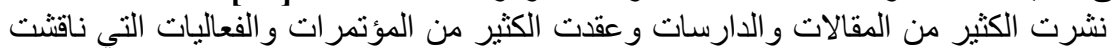

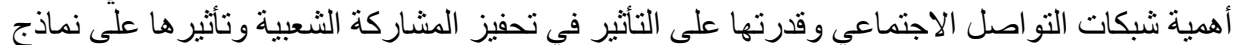

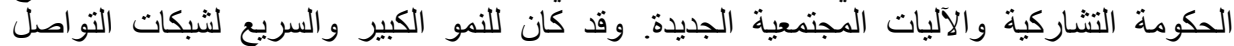

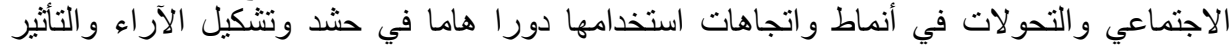

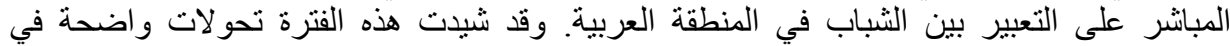

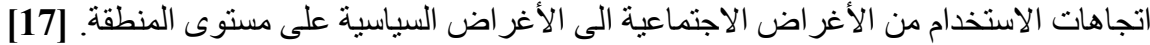

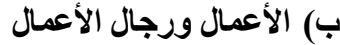

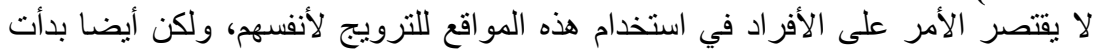

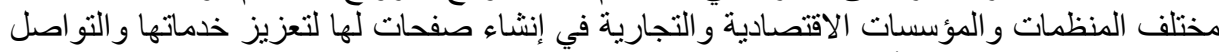

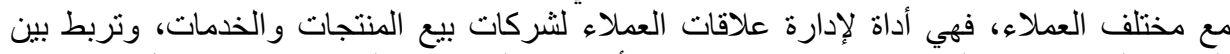

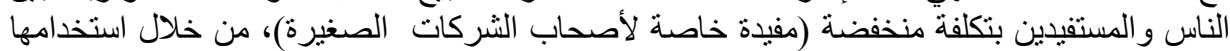

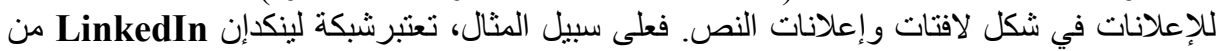

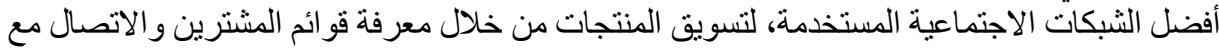

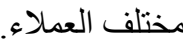

\section{ج) طبية وإدارة المعرفة الصحية}

تستخدم الثبكات الاجتماعية في إدارة المعرفة الصية الصحية للمؤسسة ونشر المعرفة الضرورية و المساعدة على تجاوز الأمر اض الجسدية و العقلية.

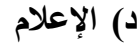

عمت التقنية جميع مناشطط الأنشطة البشرية فما كان من المؤسسات الاعلامية إلا الاستجابة

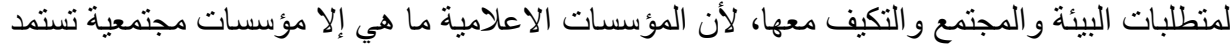

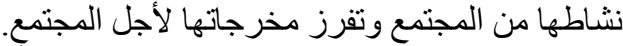

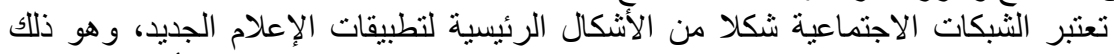

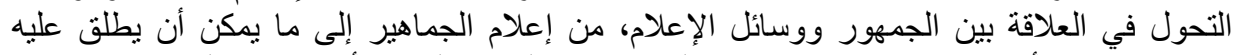

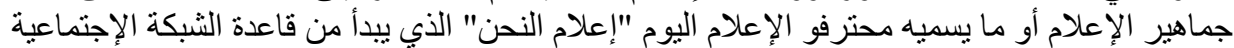

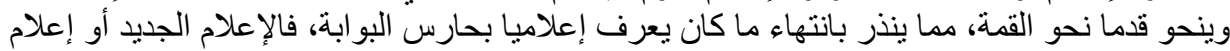

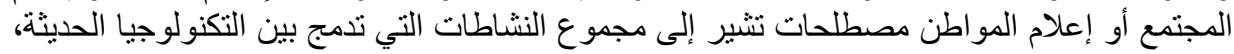

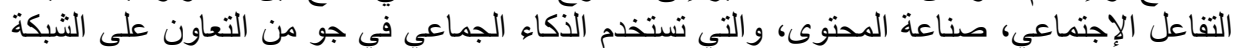

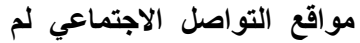
تغير طريقتنا في الاتصال فُقطّ،

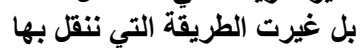
ونستهلك الأخبار. و المنتديات بجانب مو اقع عديدة مثل: الويكي، فيسبوك، التويتر ... ماي سبيس... فليكر... اليوتوب. 
إن الثورة التكنولوجية دفعت بالإعلام للعب دور هام سواء بمحاولة كسب التأييد أو الترويج ألتا لأر اء ومو اقف معينة من الأحداث التي تجري في العالم و التي شهدتها المنطقة العربية في الآونة

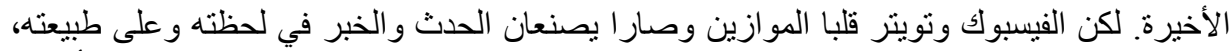

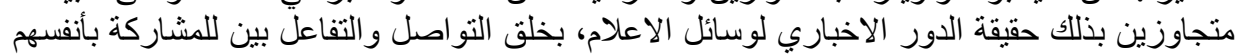

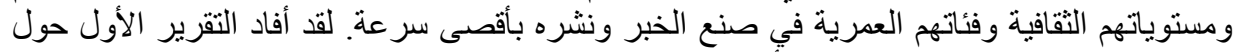

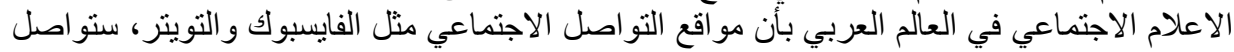

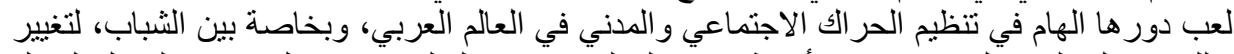

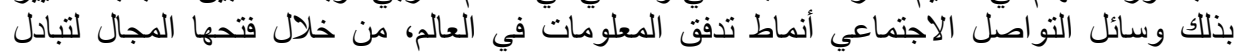

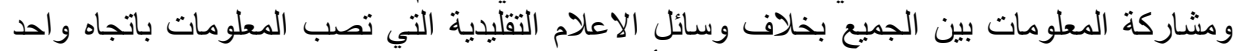

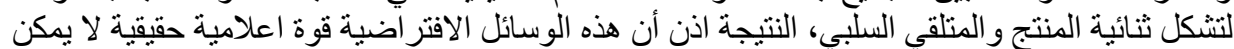

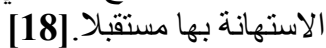

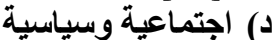

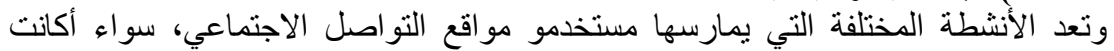

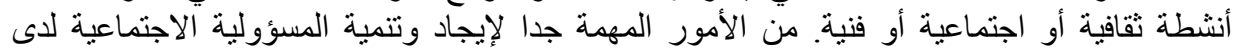

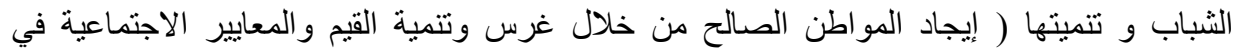

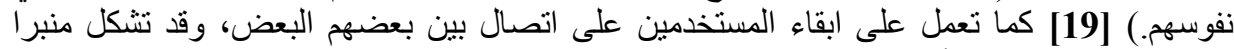
للاحتجاج و التخطيط و الأخبار.

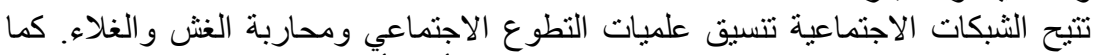

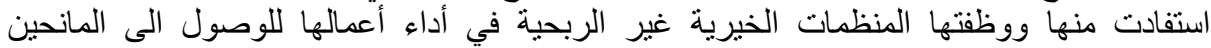

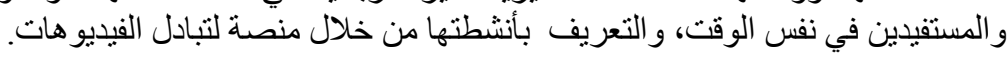

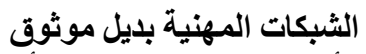

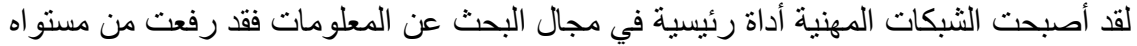

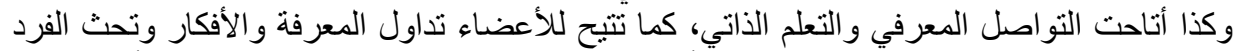

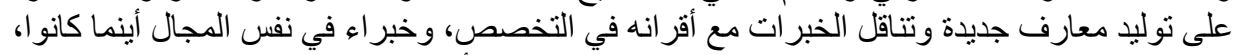

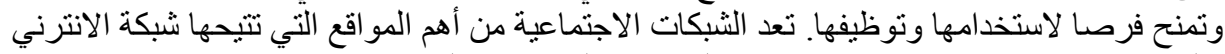

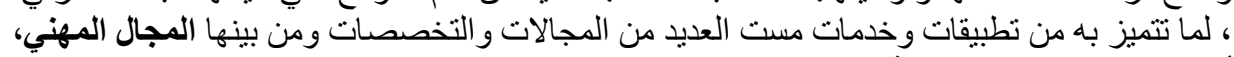

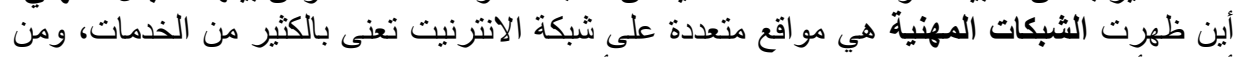

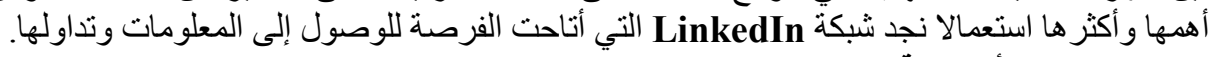

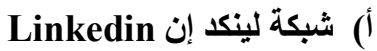

هي شبكة اجتماعية مختصة بالعمل وان والتوظيف والتجارة، نضم العديد من المحترفين

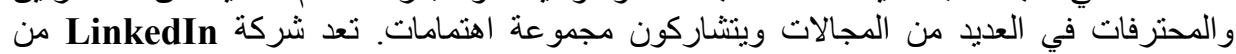

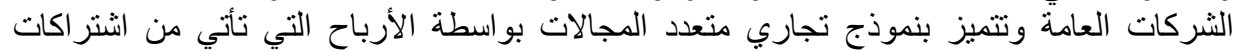

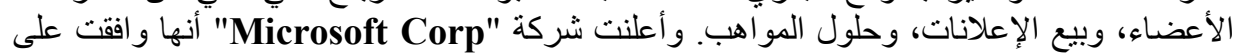

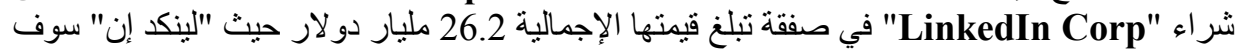

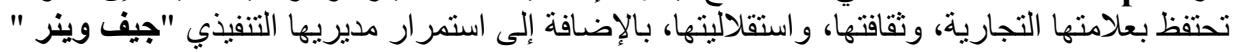

وموقع لينكإن هو شبكة اجتماعية مهنية، ففي الوقت الذي تركز فيه مواقع الثبكات في منصبه [20]

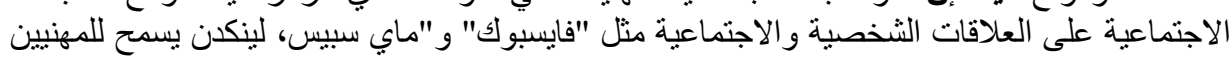

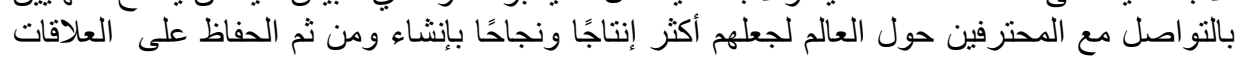

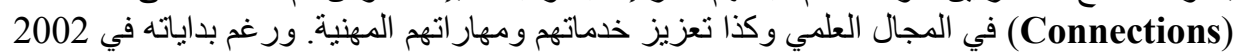




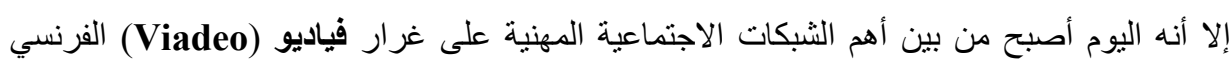
وكسينغ (Xing) الألماني ورايز (Ryze) الكوري...

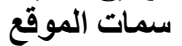

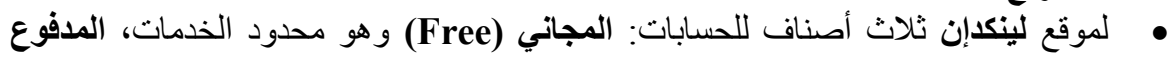
(Premium) والاحتر افي (Pro)، والاختلافات تكمن في إمكانية معرفة من اطلع على الألى

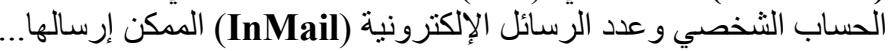

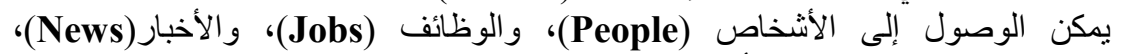

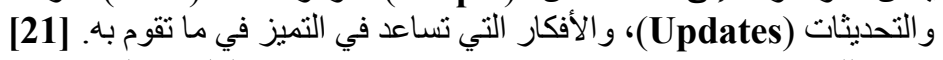

همكن للمستخدمين وضع صور (Photos) ومشاهدة الملفات الثخصية (Profiles) و الصور الخاصة بالآخرين. • يمكن للمستخدمين التعرف على على عدد الأشخاص (Connections) الذين يبحثون عنهم.

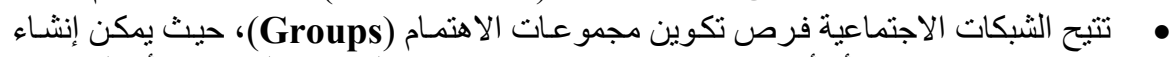

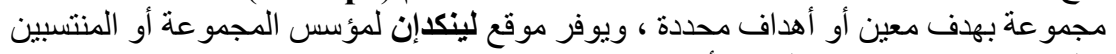

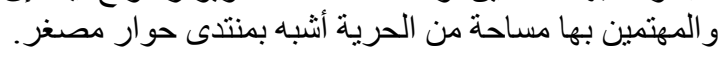

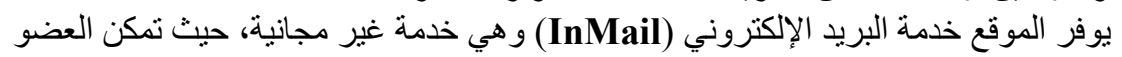

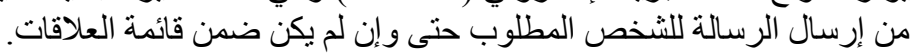

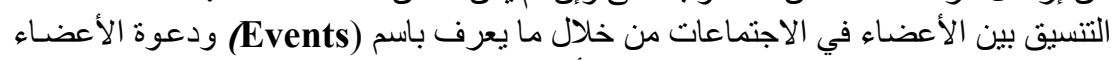

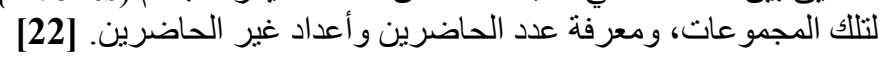

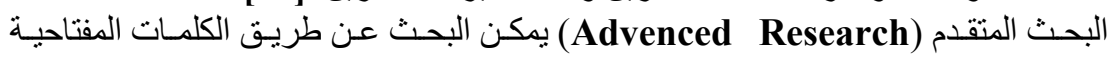

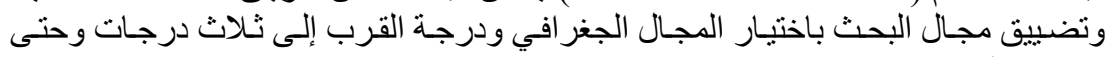
بو اسطة الوظيفة و الاختصاص. باص.

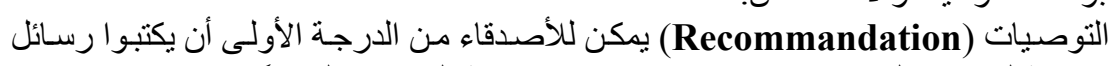

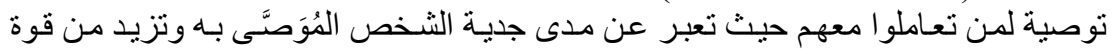
حسابه.

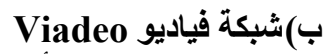

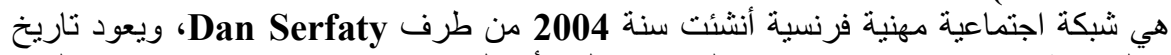

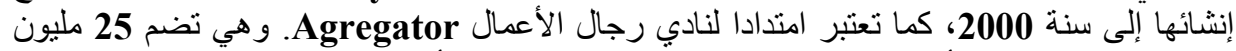

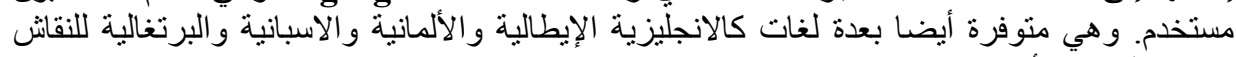

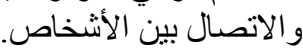

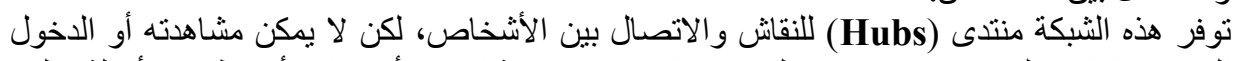

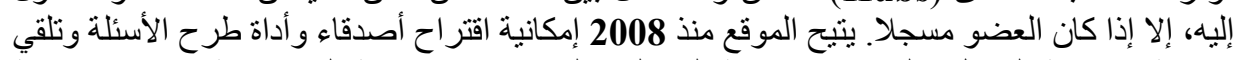

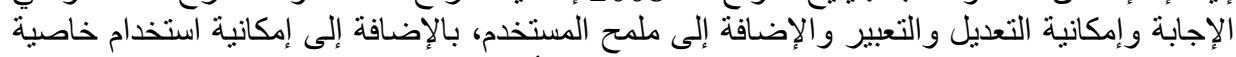

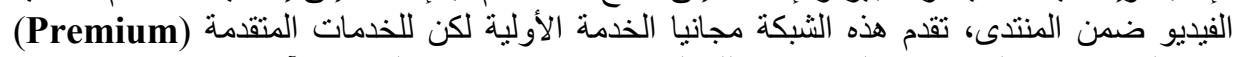
يجب الدفع. ولا تختلف سمات الموقع عن تللك الخاصة بشنبكة لئنة لينكل إن الدذكورة آنفا.

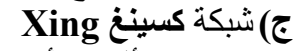

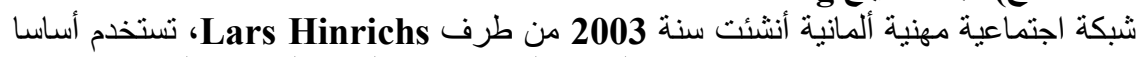

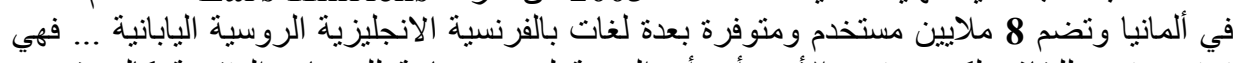

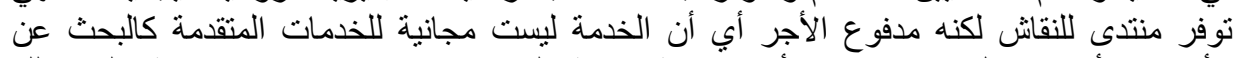

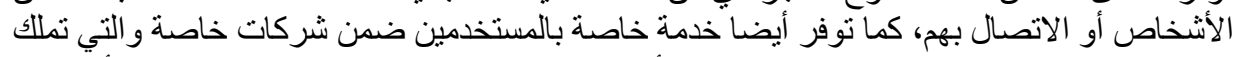

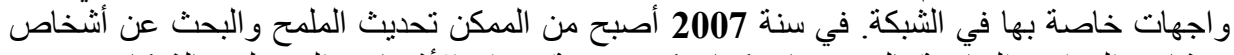

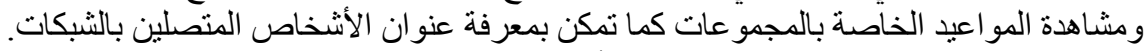
د) ولمركبلايس (WorkPlace) [23] أو (مكان العمل) 
هو فضاء مخصص و آمن للثركات أن تتصل، تتو اصل وتتعاون. منظمات من كل الأحجام تستطيع أن أن العيات تستعمل مميزات الفابسبوك العادي مثل آخر الأخبار (News Feeds)،

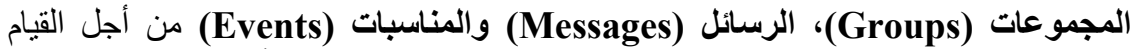

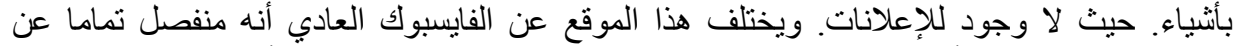

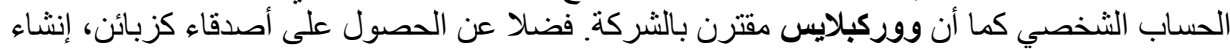

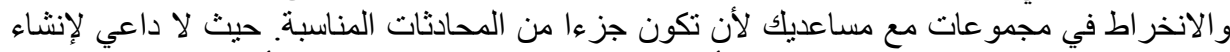

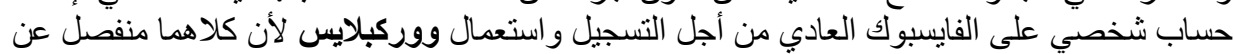

الآخر سواء الموقع أو التطبيق. [24]

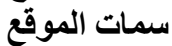

تجد الثركات أنها يمكن أن تقضي أو تحد بشكل كبير من حاجتها لأدوات التعاون الداخلية

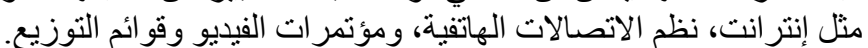

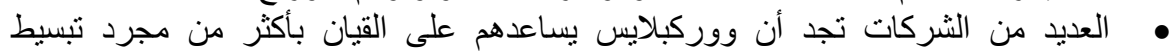

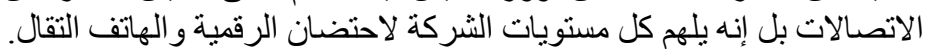

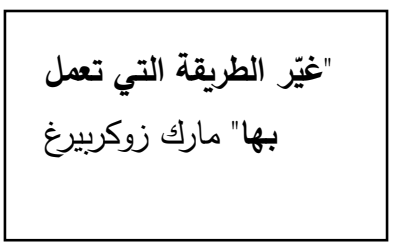

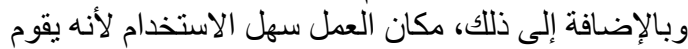

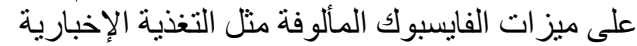
و المجمو عات و الرسائل التي لا تتطلب تدريبا.

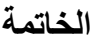
يقول المثل الإنجليزي: "تخلق النافسة الكثير من ون

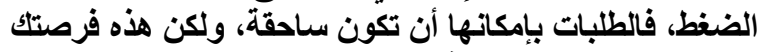

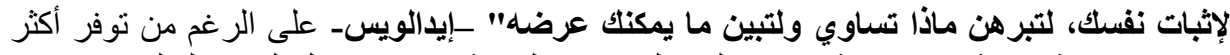

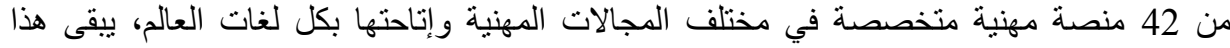

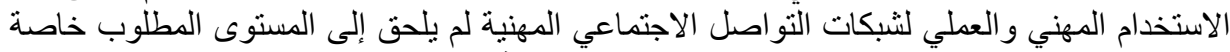

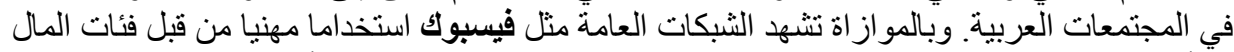

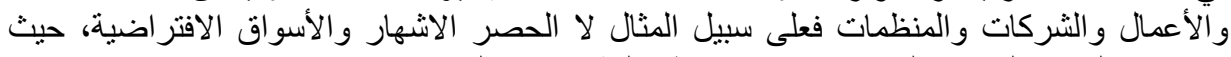

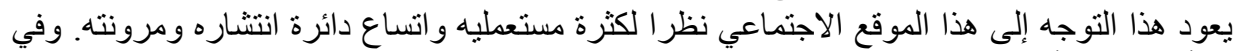

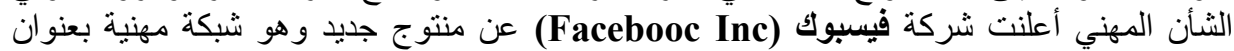
فيسبوك في العمل (Facebook At Work) أو ووركبلايس (WorkPlace) الذي من شئنس أنها أن

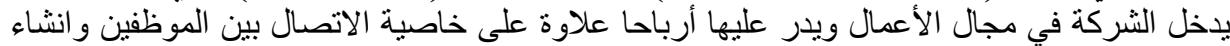

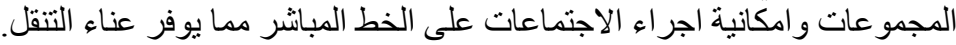

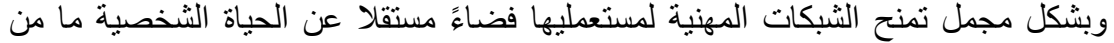

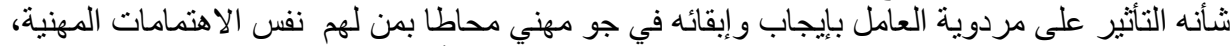
كما بساعد على التركيز في الجهد المبذول ومدة التفرغ غلائل للعمل دون أبي تشويش.

[1] رحيمة الطيب عيساني، الصراع و التكامل بين الإعلام الجديد والإعلام التقليدي، مجلة الباحث

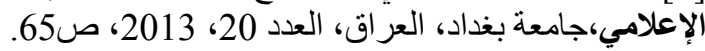
[2] عباس مصطفى الصادق ، الإعلام الجديد دراسة في مداخله النظرية، دار الثروق، عمان، 2008، [3] رضا أمين، الإعلام الجديد، دار الفجر للنشر والتوزيع، القاهرة ، ط1، 2014، 
[4]M. Briggs, "Journalism 2.0: How to survive and thrive: A digital literacy guide for the information age", J-Lab: University of Maryland, 2007, p.30 available online:

http://scholarcommons.usf.edu/cgi/viewcontent.cgi?article=1001\&context $=$ oa textbooks

[5]فيصل محمد عبد الغفار، شبكات التو اصل الاجتماعي، دار الجنادرية للنشر و التوزيع، الأردن، ط1،

[6]مروى عصام صلاح، الإعلام الإلكتروني: الأسس وآفاق المستقبل، دار الإعصار العلمي للنشر

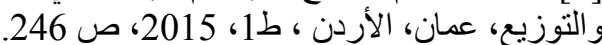

[7] زاهر راضي، "استخدام مو اقع التواصل الإن، الاجنماعي في العالم العربي"، مجلة التربية، العدد 15 ،

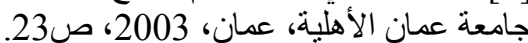

[8] Danah m. Boyd, Nicole B.Ellison , Social network sites; Definition , history and scholar ship , Journal of computer mediated communication , vol(13), issue (1),2007, p2. Available on:

http://onlinelibrary.wiley.com/doi/10.1111/j.1083-6101.2007.00393.x/full visit of 20/06/2015 at 14:04.

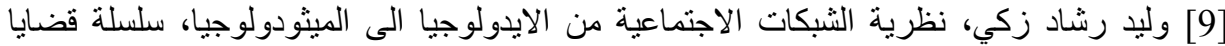

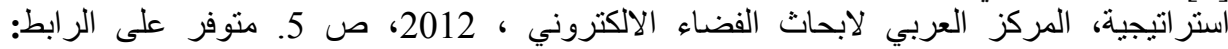

www.accr.co

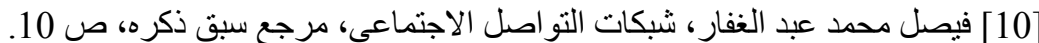

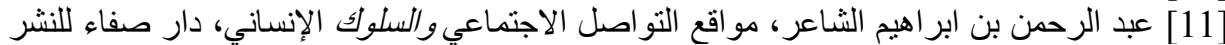

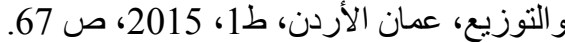

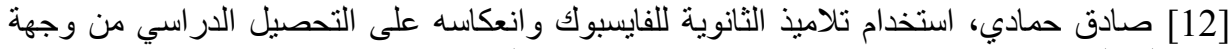

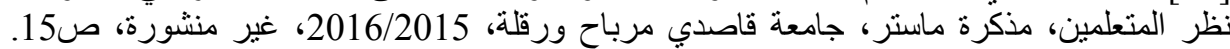

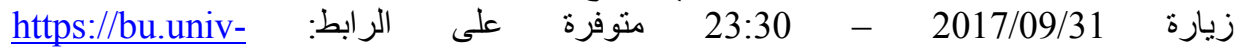
ouargla.dz/master/pdf/sadak-hamadi.pdf [13] عبد الرحمن بن ابراهيم الثاعر، مواقع التواصل الاجتماعي والسلوك الانساني، مرجع سبق

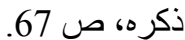
[14] سالم سعيد علي الكندي ومحمد ناصر علي الصقري، شبكات التو اصل الاجتماعي كأدوات تسويقية

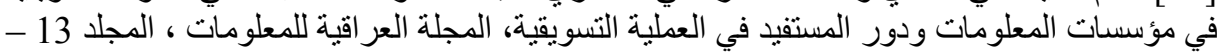

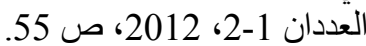

[15] عامر إبراهيم القندلجي، الإعلام والمعلومات والأنترنت، دار اليازوري للنشر والتوزيع، لبنان،

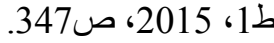

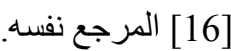

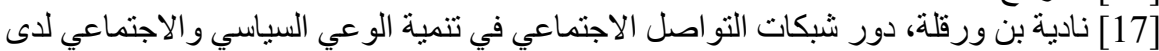

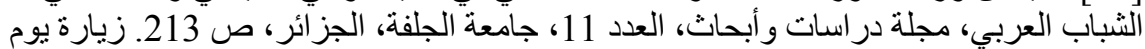
2016/10/17، 21:04، منوفر على الرابط: 
شبكات التواصل الاجتماعي بين اختصاص البنية وعمومية الاستعمال مدخل نظري

www.univ-djelfa.dz/revues/dirassat/index_htm.../Makal11_12.pdf

[18] صونية عبديش، مو اقع التو اصل الاجتماعي: الماهية، الأبعاد، الخاطر و المستقبل - مع الإنشارة

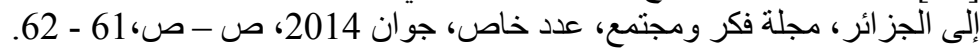

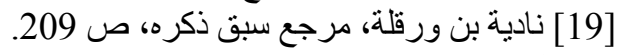

زيارة 20] https://blog.linkedin.com/2016/06/13/microsoft-and-linkedin 2016/06/18 على الساعة: 00:33

زيارة يوم: 2015/02/19 ز21] https://www.linkedin.com/about-us?trk=hb ft about

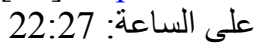

[22] وليد رشاد زكي، نظرية الثبكات الاجتماعية من الايدولوجيا الى الميثودولوجيا، مرجع سبق اليق

[23] https://workplace.fb.com/faq/ 20:40 2016/11/01

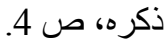

[24] http://aboutus.ft.com/2016/03/01/financial-times-to-launch-on-facebookat-work/\#axzz4O8I4rJsj visite du 25/10/2016 à $22: 00$ 